Canadian

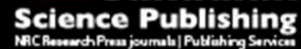

Canadian Journal of Microbiology Revue canadienne de de microbiologie

\title{
Compositional analysis: a valid approach to analyze microbiome high throughput sequencing data
}

\begin{tabular}{|r|l|}
\hline Journal: & Canadian Journal of Microbiology \\
\hline Manuscript ID & cjm-2015-0821.R2 \\
\hline Manuscript Type: & Article \\
\hline Date Submitted by the Author: & $30-$ Mar-2016 \\
\hline Complete List of Authors: & $\begin{array}{l}\text { Gloor, Gregory; The University of Western Ontario, Biochemistry } \\
\text { Reid, Gregor; The Lawson Research Institute }\end{array}$ \\
\hline Keyword: & $\begin{array}{l}\text { microbiome, compositional data, correlation, multivariate statistics, } \\
\text { multiple test correction }\end{array}$ \\
\hline
\end{tabular}

SCHOLARONE ${ }^{\text {IM }}$

Manuscripts 
Compositional analysis: a valid approach to analyze microbiome

high throughput sequencing data

7 1. Department of Biochemistry, Western University, London, Ontario, Canada

2. Canadian Center for Human Microbiome and Probiotic Research, Lawson Health Research Institute, London, Ontario, Canada

3. Departments of Microbiology and Immunology, and Surgery, Western University,

15 * Address for Correspondence: Gregory B. Gloor, E-mail: ggloor@uwo.ca 


\section{Abstract}

A workshop held at the 2015 annual meeting of the Canadian Society of

26 Microbiologists highlighted compositional data analysis methods, and the importance of

27 exploratory data analysis, for the analysis of microbiome datasets generated by high

28 throughput DNA sequencing. A summary of the content of that workshop, a review of

29 new methods of analysis, and information on the importance of careful analyses are

30 presented herein. The workshop focussed on explaining the rationale behind the use of

31 compositional data analysis, and a demonstration of these methods for the examination

32 of two microbiome datasets. A clear understanding of bioinformatics methodologies and

33 the type of data being analyzed is essential given the growing number of studies

34 uncovering the critical role of the microbiome in health and disease, and the need to

35 understand alterations to its composition and function following intervention with fecal

36 transplant, probiotics, diet and pharmaceutical agents.

40 Key Words: microbiome, compositional data, correlation, multiple test correction 


\section{Introduction}

51 Human microbiome studies have shown a major link between microbial

52 composition and health and disease and dysbiosis (Fremont et al. 2013; Lourenço et al.

53 2014; Urbaniak et al. 2014). High throughput DNA sequencing methodologies have

54 made this possible, along with breakthroughs in culturing techniques. The former has

55 used approaches such as 16S rRNA gene sequencing, metagenomics, transcriptomics

56 and meta-transcriptomics, leading to vast datasets that must be simplified and analyzed

57 (Di Bella et al. 2013). Indeed, each sample may have tens of thousands to millions of

58 sequence reads associated with it, and the entire dataset across all samples can easily

59 exceed many hundreds of millions of reads. Such has been the rapidity of these

60 developments that some studies appear to have been published using methods that are

61 potentially. The result can be papers with serious deficiencies that are publicized as

62 major advances or breakthroughs (Reardon 2013), when in some cases the data are far

63 from sufficient for such claims. We will examine the evidence for one of these papers

64 below (Hsiao et al. 2013).

65 Data for microbiome analysis are collected by the following general workflow.

66 The sample (swab, stool, saliva, urine or other type) is collected, the DNA is isolated

67 and used in a polymerase chain reaction with primers specific to one or more variable

68 regions of the $16 \mathrm{~S}$ rRNA gene. It is also possible to target other conserved genes such

69 as the cpn60 gene (Schellenburg et al. 2009). However, analysis problems are the 
70 same regardless of the amplification target chosen, and Walker et al. (2015) present a

71 good summary of how choices taken upstream of data analysis affect the results.

72 Following amplification, a random sample of the product is used to make a sequencing

73 library, and it is common to multiplex many samples in the library. A small aliquot of the

74 library is processed on the high throughput DNA sequencing instrument. As outlined

75 below, this workflow imposes constraints on the resulting data.

76 It should be recognized that the investigator is sequencing a random sample of

77 the DNA in the library, which is itself a random sample of the DNA in the environment.

78 Thus, it is important to ensure that any analysis takes this random component into

79 account (Fernandes et al. 2013).

80 Perhaps less obvious is that the number of sequencing reads obtained for a

81 sample bears no relationship to the number of molecules of DNA in the environment,

82 because the number of reads obtained for a sample is determined by the capacity of the

83 instrument. For example, the same library sequenced on an Illumina MiSeq or HiSeq

84 would return approximately 20 million or 200 million reads. That there is no information

85 in the actual read numbers per sample is implicitly acknowledged by the common use of

86 'relative abundance' values for analysis of microbiome datasets. Such datasets are

87 referred to as compositional and there is a long history of the development of proper

88 analysis techniques for such data in other fields (Pawlowsky-Glahn et al. 2015).

89 Compositional data is a term used to describe a dataset in which the parts in

90 each sample have an arbitrary or non-informative sum (Aitchison 1986), such as data

91 obtained from high throughput DNA sequencing (Friedman and Alm 2012, Fernandes et

92 al. 2013, 2014). These data have long been known to be problematic (Pearson 1896), 
93 and we now understand that multivariate data analysis approaches such as ordination

94 and clustering and univariate methods that measure differential abundance are invalid

95 (Aitchison 1986, Warton et al. 2012, Friedman and Alm 2012, Fernandes et al. 2013

96 Pawlowsky-Glahn et al. 2015).

97 The essential problem is illustrated in Figure 1 where we set up an artificial

98 example and count the number of molecules in the environment. We allow one part

99 (shown as solid black) to increase 10-fold between samples 1 and 2, while the

100 abundance of the other 49 parts (in open circles) remain unchanged. The proportion

101 panel shows how the data are distorted when we convert it to relative abundances or

102 proportions, or as happens when the sequencing instrument imposes a constant sum.

103 The black part still appears to become more abundant, although it is less than a 10 -fold

104 change. However, the 49 other parts appear to become less abundant. This property

105 leads to the negative correlation bias observed in compositional data, and renders

106 invalid any type of correlation or covariance based analysis such as correlation

107 networks, principle component analysis, and others (Pearson 1896, Aitchison 1986).

108 Note that this distortion will also lead to false univariate inferences as well (Fernandes

109 et al. 2013,2014).

110 The original issue with compositional data identified by Pearson (1896) was that

111 of spurious correlation. That is, two or more variables can appear to be correlated

112 simply because the data are transformed to have a constant sum. Spurious correlation

113 also causes the correlations observed in these data to depend on the membership of

114 the sample. For example, consider the simple case of three samples ( $a, b$ and $c)$ with 
115 four taxonomic variables measured to have the following absolute counts in three

116 environmental samples (i.e., samples are in rows, taxa are in columns):

117

$$
a b c=\left[\begin{array}{cccc}
470 & 66 & 839 & 751 \\
541 & 569 & 787 & 512 \\
167 & 906 & 959 & 504
\end{array}\right], \operatorname{cor}(a b c)=\left[\begin{array}{cccc} 
& -0.68 & -\mathbf{0 . 9 9} & 0.36 \\
-0.77 & & \mathbf{0 . 5 9} & -0.93 \\
\mathbf{- 0 . 3 0} & -\mathbf{0 . 3 7} & & -0.25 \\
0.55 & -0.95 & 0.62 &
\end{array}\right]
$$

The Pearson correlation for the numerical values is in the upper triangle of the

119 right hand matrix, and we see that taxon 1 and taxon 3 have a near perfect negative

120 correlation of -0.99 (shown in bold), and taxon 2 and taxon 3 have a positive correlation

121 of 0.59 . The lower triangle on the right hand matrix shows the Pearson correlation

122 values that are found when these are converted to relative abundances by dividing by

123 the total sum of counts in each sample. Now, the correlations between the same taxa

124 have changed. The correlation between 1 and 3 is now moderately negative at -0.30 ,

125 and between 2 and 3 is now -0.37 . Thus, the correlation observed in compositional data

126 is not the same as the correlation for the counts, and the correlations measured can

127 even change sign.

128 There is a further complication: the correlations observed in compositional data

129 depend on the membership in the sample. So, for example, when the last value is

130 dropped from each sample, the correlations between taxa 1 and 2 is positive (0.43), and

131 the correlation between 2 and 3 is even more strongly negative at -0.79 . Thus,

132 correlation determined from compositional data has the potential to be wildly wrong, and

133 normal approaches to determine correlation cannot be used (Friedman and Alm 2012,

134 Lovell et al. 2015, Kurtz et al. 2015). It is worth noting that any method of determining

135 correlation (including Spearman, Kendall, etc) will suffer from the same problems. Thus

136 the current tools used to examine the analysis goals give results that may be 
137 inconsistent, difficult to interpret and in many cases completely wrong (Filmoser et al.

138 2009, Friedman and Alm 2012, Fernandes et al 2013, Fernandes et al. 2014, Lovell et

139 al. 2015, Kurtz et al. 2015).

140 The essential first step of proper compositional data analysis is to convert the

141 relative abundances of each part, or the values in the table of counts for each part, to

142 ratios between all parts. This can be accomplished in several ways (Aitchison 1986), but

143 the most widely used and most convenient for our purposes is to convert the data using

144 the centred log-ratio (clr) transformation. So if $X$ is a vector of numbers that contains $D$

145 parts:

$146 \mathrm{X}=\left[\mathrm{x}_{1}, \mathrm{x}_{2}, \ldots \mathrm{x}_{\mathrm{D}}\right]$,

147 the centered log-ratio of $X$ can be computed as:

$148 \mathrm{X}_{\mathrm{clr}}=\left[\log \left[\mathrm{x}_{1} / \mathrm{gx}_{\mathrm{X}}\right], \log \left[\mathrm{x}_{2} / \mathrm{gx}_{\mathrm{x}}\right], \ldots \log \left[\mathrm{x}_{\mathrm{D}} / \mathrm{gx}_{\mathrm{X}}\right]\right.$

149 where $g_{X}$ is the geometric mean of all values in vector $X$ (Aichison 1986). This

150 simple transformation renders valid all standard multivariate analysis techniques

151 (Aitchison 1986, van den Boogaart 2013, Pawlowsky-Glahn et al. 2015), and as shown

152 in the Ratios panel of Figure 1, can reconstitute the shape of the data so that univariate

153 analyses are also more likely to be valid. This transformation is also the starting point

154 for essentially all compositional data analysis ( $\mathrm{CoDa}$ ) based assessments of the

155 datasets.

156 A CoDa approach would be robust if microbiome datasets were not sparse, that

157 is, they did not contain any 0 values. However a frequent criticism of the CoDa

158 approach is that the geometric mean cannot be computed if any of the values in the

159 vector are 0 . It is here we reiterate that our data represent the counts per taxon through 
160 the process of random sampling (Fernandes et al. 2013, 2014). Thus, some 0 values

161 could arise simply by random chance, while others arise because of true absence of the

162 taxon in the environment. Fortunately, we can couple Bayesian approaches to estimate

163 the likelihood of 0 values with the compositional analysis approach (Fernandes et al.

1642013,2014 , Gloor et al. 2016). With this paradigm we dispose of taxa with 0 counts in

165 all or most samples (Palarea-Albaladejo and Martin-Fernandez 2015), and assign an

166 estimate of the likelihood of the 0 being a sampling artifact to the remainder. When

167 performing univariate tests or correlation analyses, it is often convenient to keep many

168 such estimates of 0 and to determine the expected value of test statistics to reduce

169 false positive inferences (Friedman and Alm 2012, Fernandes et al. 2013, Fernandes et

170 al. 2014).

171 Microbiome analysis tools that account for compositional data

172 Fortunately, the compositional data analysis problem of microbiome datasets is starting

173 to be examined by several groups and there are now an increasing number of tools

174 available as outlined below.

175 These tools can be applied to address three major objectives of many microbiome 176 analyses:

177 1. Do the data show any structure? That is, do the data partition into groups?

178 2. What is the difference between groups? This can be between groups identified 179 beforehand, or following the exploratory data analysis.

180 3. What is the correlation structure of the taxonomic groups? Do any of these taxa $181 \quad$ correlate with the metadata? 
182 These analyses are usually done using either the mothur (Schloss et al. 2009) or the

183 QIIME (Kuczynski et al. 2012) aggregated toolsets, containing approaches adapted

184 from the field of ecology. However, the use of an analysis paradigm based on

185 compositional data analysis (Aitchison 1986), or CoDa, offers a number of advantages

186 over these tools, as explained below.

187 The first objective is to determine if there is structure in the dataset. In the

188 microbiome field this is generally described as beta-diversity analysis. Beta-diversity as

189 currently used requires a distance or dissimilarity measure, and popular ones include

190 the unweighted or weighted Unifrac distance metrics (Lozopone and Knight 2005) or the

191 Bray-Curtis dissimilarity metric. These methods are included in both the mothur and

192 QIIME toolkits. The distance metrics from these tools can be used to generate Principle

193 Co-ordinate (PCoA) plots that can be used to assess similarities and differences

194 between samples and groups. Unfortunately, distance-based tools can confuse location

195 (difference) and dispersion (variance) effects (Warton et al. 2012), and so additional

196 approaches based on a compositional paradigm should be used for exploratory data

197 analysis.

198 The CoDa analysis analog to PCoA is a principle component analysis (PCA) of

199 center-log ratio transformed data that has been modified to either remove taxa with 0

200 observed counts, or to adjust 0 values to an estimated value (Palarea-Albaladejo and

201 Martin-Fernandez 2015). PCA has the advantage of being a more interpretable metric

202 than PCoA, since it directly assesses the variance in the data and because both the

203 locations of the samples and the contribution of each taxon to the total variance can be

204 shown on the so-called compositional biplot (Aitchison and Greenacre 2002). The ability 
205 to examine variation of both the samples and the taxa on the same plot provides

206 powerful insights into which taxa are compositionally associated and which taxa are

207 driving (or not) the location of particular samples. Thus, the biplot can serve as a

208 summary of the entire dataset, and it is up to the investigator to attach numerical

209 significance to the qualitative results observed. The example usage of compositional

210 biplots is explained in detail below.

211 The second major objective is often to determine which taxa are driving the

212 difference observed between groups. Several methods are in widespread use to assess

213 the difference in abundance of taxa between groups. These include microbiome specific

214 methods such as Metastats (White et al. 2009) or LEfSe (Segata et al. 2011), and more

215 general t-tests or nonparametric tests. However, all use as input a table of proportional

216 abundances. As shown in Figure 1, examination of proportions can result in a gross

217 distortion of the data, such that some taxa can appear to change in abundance when

218 measured by proportion, when in fact, their true abundance in the environment may be

219 unchanged. This effect can be ameliorated by the center-log ratio transformation.

220 There are two approaches that assess differential abundance in a compositional

221 data analysis framework. The simplest approach is the ANCOM tool (Mandal et al.

222 2015), which assesses statistical significance on log-ratio transformed data. This is

223 more robust than both traditional t-tests and more sophisticated approaches such as

224 zero-inflated Gaussian methods. It should be noted that the software is not currently

225 deposited into a public repository, and that the 0 -replacement value used is fixed in the

226 software. 
A slightly more complex approach is used by the ALDEx2 package, available

228 from Bioconductor (Fernandes et al 2013, Fernandes et al 2014). Like ANCOM,

229 ALDEx2 centre log-ratio transforms the data prior to the assessment of statistical

230 significance, however ALDEx2 differs greatly in how values of 0 are handled. ALDEx2

231 estimates a large number of possible values for 0 (and any other count for a taxon in a

232 sample), conducts significance tests on all estimated values, and takes the average

233 significance test value as the most representative for that taxon. In essence, ALDEx2

234 determines which taxa are significantly different between groups after accounting for the

235 random sampling that occurs when the DNA is extracted and loaded onto the

236 sequencing instrument. In either case, both ANCOM and ALDEx2 explicitly

237 acknowledge the multivariate compositional nature of the data, and control for false

238 positive identifications much better than do the usual approaches.

239 The third objective is to determine if there are taxa in the dataset with correlated

240 abundances. As noted above, spurious correlation is a very large problem in

241 microbiome datasets. Therefore, analyses that report correlations using traditional

242 methods, such as Pearson's or Spearman's correlations, Kendall's Tau or Partial

243 correlations are likely to be wrong (Friedman and Alm 2012, Lovell et al. 2015, Kurtz et

244 al 2015). However, there are a number of approaches that use a compositional data

245 analytic approach to correlation. In a compositional approach, the variance between

246 ratios of two taxa should be 0 or nearly so for two taxa to be counted as correlated

247 (Aitchison 1986, Lovell et al. 2015). The difficulty comes when placing this approach

248 into a familiar null hypothesis test framework, or when applying a consistent scale to the

249 measure. The simplest approach is to calculate the phi statistic for two taxa $\mathrm{X}$ and $\mathrm{Y}$, 
250 which is the $\operatorname{var}(\log (X / Y) / \operatorname{var}(\log (X)($ Lovell et al. 2015), where $\log ()$ is meant to imply

251 the clr values of $\mathrm{X}$ or $\mathrm{Y}$. This measure has the advantage of being easily calculated and

252 of strictly enforcing the compositional data analysis approach. The SparCC method

253 (Friedman and Alm, 2012) uses Bayesian estimates of the value of $X$ and $Y$ but

254 calculates a mean value of a measure similar to the concordance correlation coefficient.

255 The SPIEC-EASI approach (Kurtz et al. 2015) uses clr-transformed values and infers a

256 graphical model under the assumption of a sparse correlation network. Both of the latter

257 approaches make strong assumptions about the sparsity of the data, and so are less

258 rigorous for estimating correlations in compositional data than is the calculation of phi.

259 However, they both offer the advantage of using a full or partial Bayesian approach,

260 which is generally more powerful than point-estimate based approaches.

261 Application of CoDa to Two Case Studies

262 Having introduced the issue of compositional data analysis, we now present the 263 results of two worked examples presented at the Bioinformatics Workshop was held on

264 June 16, 2015 in Regina at the Annual Scientific Meeting of the Canadian Society of

265 Microbiologists. This illustrates how these approaches can be applied to two different

266 16S rRNA gene sequencing datasets from the recent literature. A full description of the

267 methodology, the datasets and the code used to generate the figures is given in the

268 Supplementary file workshop.Rnw (Gloor 2016). Downloading and running this file in R

269 (R Core Team 2015) or RStudio will generate the associated workshop.pdf. The .Rnw

270 document contains both the code and annotation for the code, and the .pdf document

271 contains the code and the resulting figures. 
The first worked example is a vaginal microbiome dataset. This dataset is from

273 an experiment that examined the effect of treating women suffering from bacterial

274 vaginosis (BV) with antibiotics and placebo or antibiotics plus a probiotic supplement

275 (Macklaim et.al, 2015). For this example, we extracted only the 'before' (samples

276 labeled as BXXX) and 'after' ( $\mathrm{XXXX)}$ treatment samples, which were further identified by

277 their Nugent status, a Gram stain scoring system that acts as a rough indicator of

278 whether the subject had BV or was healthy (normal, $n$ ), or whose status was

279 indeterminate (labeled as ' $\mathrm{l}$ ' for intermediate). In addition, individual taxa were

280 aggregated to genus level using QIIME (Kuczynski et al. 2012), except for Lactobacillus

281 iners and Lactobacillus crispatus, which remained as separate species in the tables.

282 This relatively simple dataset will be used to introduce and explain the CoDa analysis

283 methods.

284 The compositional biplot is the essential initial tool for exploratory compositional

285 data analysis and replaces ordinations based on Unifrac or Bray-Curtis metrics.

286 Compositional biplots are principle component plots of the singular value decomposition

287 of the data. This approach displays the major axes of variance (or change) in a dataset

288 (Aitchison and Greenacre 2002). Properly made and interpreted, these plots summarize

289 all the essential results of an experiment. However, it should be remembered that they

290 are descriptive and exploratory, not quantitative. Quantitative tools can be applied later

291 to support the conclusions derived from the biplot.

292 For simplicity, we filtered the dataset to include only those taxa that were at least

$2930.1 \%$ abundant in any sample. One of the desirable properties of compositional data

294 analysis is that subsets of the dataset are expected to give essentially the same answer 
295 as the entire dataset for the taxa in common between the whole and the subset dataset

296 (Aitchison 1986).

297 Figure 2 shows the compositional biplot for this dataset along with the associated

298 scree plot that displays the percentage of variance explained by each sample or

299 component. The sample names (labeled in red for BV, blue for Normal or purple for

300 Intermediate) illustrate the variance of the samples, and the taxa values (represented by

301 the black rays) illustrate the variance between the taxa. In fact, the length of the arrow

302 for each taxon is proportional to the standard deviation of the ratio of each taxon to all

303 other taxa. There are many interpretation rules for biplots of compositional data

304 (Aitchison and Greenacre 2002), but these rules are dependent on remembering that

305 only the ratios between taxa can be examined. Thus, the links between the tips of the

306 rays, or between samples contain the most information. Keeping this in mind, we can

307 see the following:

308 First, the proportion of variance explained in the first component is very good,

309 being $47 \%$, then falling to $13 \%$ on component 2 , and decreasing rapidly thereafter. This

310 indicates that the major difference between samples can be captured in essentially one

311 direction along component 1 . While the amount of variance explained on the first

312 component is relatively large in this dataset, a rule of thumb is that PCA plots that

313 display less than $80 \%$ of the variance on the first two components are not necessarily

314 accurate projections of the data. Thus, some of the quantitative results are expected to

315 be somewhat different than is displayed in the qualitative PCA projection.

316 Second, the longest link from the center to a taxon is the one to Lactobacillus

317 iners. This indicates that the ratio of this taxon to all others is the most variable across 
318 all samples. Likewise, the shortest link is to Gardnerella, implying that the ratio of this

319 taxon to all others is the least variable.

320 Third, the longest link is between L. iners and Leptotrichia (Sneathia). This

321 means we can infer that these two taxa likely have the strongest reciprocal ratio

322 relationship. That is, when one becomes more abundant relative to everything else, the

323 other becomes less abundant relative to everything else.

$324 \quad$ Fourth, the shortest link observed in the plot is between Megasphaera and

325 BVAB2. From this we conclude that the ratio of these two taxa is relatively constant

326 across all samples. That is, their ratio abundance is highly correlated. These two taxa

327 should be seen to have a low value of phi, but we must keep in mind the limit of the

328 projection of the data.

Fifth, the link between Prevotella and Lactobacillus crispatus passes directly

330 through Atopobium. This indicates that these three taxa are linearly related. In this case,

331 it is clear when L. crispatus increases, the other two will decrease. Likewise, this

332 property can be extended to any linear relationships containing three or more links.

333 Sixth, the link between L. iners and Megasphaera, and the link between

334 Leptotrichia (Sneathia) and Lactobacillus cross at approximately $90^{\circ}$. The cosine of the

335 angle approximates the correlation between the connected log ratios. Thus, we can

336 conclude that the abundance relationship between the former pair of taxa is poorly

337 correlated with that of the latter two taxa. In other words, these two pairs vary

338 independently in the dataset.

339 Some samples (A312_bv, B312_i, A282_n at the bottom), are tightly grouped,

340 indicating that they contain similar sets of taxa at similar ratio abundances. We can see 
341 from the biplot that these samples contain an abundance of Lactobacillus and are

342 depleted in Leptotrichia (Sneathia). Furthermore, we can see that the samples divide

343 into two fairly clear groups, with most of the before or "B" samples on the left, and most

344 of the after or "A" samples on the right. We further observe that the majority of the B

345 samples are colored red indicating a diagnosis of $\mathrm{BV}$, and the majority of the A samples

346 are colored blue indicating a diagnosis of non-BV.

347 The result of the biplot suggested that there were two main groups that could be

348 defined with this set of data. With a few exceptions, there appears to be a fairly strong

349 separation between the samples containing a majority of Lactobacillus sp., and those

350 lacking them. We can explore this by performing an unsupervised cluster analysis on

351 the log-ratio transformed data. In traditional microbiome evaluation methodologies,

352 clustering is based on the weighted or unweighted unifrac distances or on the Bray-

353 Curtis dissimilarity metric, for example see the standard workflow in QIIME (Kuczynski

354 et al. 2012). These metrics are much more sensitive to the abundance of community

355 members than is the Aitchison distance used in compositional data analysis (Martin

356 Fernandez 1998). Thus, here we used the Aitchison distance metric that fulfills the

357 criteria required for compositional data. In particular, by using a compositional approach,

358 it is appropriate to examine a defined sub-composition of the data (i.e., a subset of the 359 taxa).

$360 \quad$ The results of unsupervised clustering of the dataset are shown in Figure 3.

361 Again, it is important to remember that all distances are calculated from the ratios

362 between taxa, and not on the taxa abundances themselves. For this figure, we used the

363 ward.D2 method which clusters groups together by their squared distance from the 
364 geometric mean distance of the group. There are many other options, and the user

365 should choose one that best represents the data, although Ward.D and Ward.D2 are

366 usually the most appropriate (Martin-Fernandez 1998).

$367 \quad$ The cluster analysis supports the results of the biplot and shows the split

368 between two types of samples rather clearly. Samples containing an abundance of

369 Lactobacillus sp. are grouped together on the right, and samples with an abundance of

370 other taxa are grouped together on the left. The cluster analysis helps explain and

371 clarify the compositional biplot. For example, the four samples in the middle lower part

372 of the biplot in Figure 2 labelled A/B312 and A/B282, group together in both the biplot

373 and the cluster plot. These samples are atypical for both the $\mathrm{N}$ and BV groups,

374 containing substantially more of the Lactobacillus taxon, and somewhat more of the

375 taxa normally found in BV than in the other $\mathrm{N}$ samples. Based on these two results it

376 would be appropriate to exclude these four samples from further analysis because of

377 their atypical makeup.

378 Next, a univariate comparison between the B and A groups was performed. For

379 simplicity of coding, we kept the outlier samples, but the reader is encouraged to

380 remove them and see how the results change. For this, we used the ALDEx2 tool

381 (Fernandes et al. 2013, 2014) that incorporates a Bayesian estimate of taxon

382 abundance into a compositional framework, with the results shown in Table 1 and the

383 effect plot (Gloor et al. 2016) shown in Figure 4. Of note, ALDEx2 examines differential

384 abundance by estimating the measurement error inherent in high throughput DNA

385 sequencing experiments, including the measurement error associated with 0 count taxa, 
386 and uses the assumptions of compositional data analysis to normalize the data for the

387 differing number of reads in each sample (Fernandes et al. 2013, Lovell et al. 2015).

388 When interpreting these results, it is important to remember that we are actually

389 examining ratios between values, rather than abundances. Thus, we are examining the

390 change in abundance of a taxon relative to all others in the dataset. The user should

391 also remember that all values reported are the means or medians over the number of

392 Dirichlet instances as given by the mc.samples variable in the aldex.clr function and

393 explained more fully in the supplementary material and the original papers (Fernandes

394 et al. 2013, 2014).

395 In the examples given in Table 1, we filtered to show only those taxa where the

396 expected Bejamini-Hochberg (1995) adjusted $P$ value was less than 0.05, meaning that

397 the expected likelihood of a false positive identification per taxon is less than $5 \%$, with

398 the actual value per taxon given in the wi.eBH column. Using $L$. iners, we note that the

399 absolute difference between groups can be up to -2.25 . Thus, the absolute fold change

400 in the ratio between $L$. iners and all other taxa between groups for this organism is on

401 average 4.76 fold $\left(1 / 2^{-2.25}\right)$ : being more abundant in the $A$ samples than in the $B$

402 samples. However, the difference within the groups (roughly equivalent to the standard

403 deviation) is even larger, giving an effect size of -0.79 . Thus, the difference between

404 groups is less than the variability within a group, a result that is typical for microbiome 405 studies.

406 These quantitative results are largely congruent with the biplot, which showed

407 that the taxa represented here were the ones that best explained the variation between

408 groups, and that the Leptotrichia (Sneathia) and Lactobacillus taxa were not contributing 
409 to the separation of the two large groups and so would not be expected to be

410 significantly different, despite being highly variable.

411 The left panel of Figure 4 shows a plot of the within (diff.win) to between (diff.btw)

412 condition differences, with the large black dots representing those that have a $\mathrm{BH}$

413 adjusted $P$ value of 0.05 or less. Taxa that are more abundant than the mean in the $B$

414 samples have positive $y$ values, and those that are more abundant than the mean in the

415 A samples have negative y values. These are referred to as 'effect size' plots, and they

416 summarize the data in an intuitive way (Gloor et al. 2015). The grey lines represent the

417 line of equivalence for the within and between group values. Small black dots represent

418 taxa that are less abundant than the mean taxon abundance: here it is clear that the

419 abundance of rare taxa, are generally difficult to estimate with any precision.

420 The middle plot in Figure 4 shows a plot of the effect size vs. the $\mathrm{BH}$ adjusted $\mathrm{P}$

421 value, with a strong correspondence between these two measures. In general, an effect

422 size cutoff is preferred because it is more robust than $\mathrm{P}$ values. The right plot in this

423 figure shows a volcano plot for reference.

$424 \quad$ Finally, we can determine which taxa are most correlated or compositionally

425 associated. As noted above, correlation is especially problematic, and the only way to

426 avoid false positive associations is to identify those taxa that have constant or nearly

427 constant ratios in all samples: this is the underlying basis of the phi measure (Lovell et

428 al. 2015). In the example shown in the supplementary material, we calculate the mean

429 phi using the same philosophy as outlined above for univariate statistical tests.

431 identify those pairs of taxa that have a near constant ratio abundance across all 
432 samples. Applying this approach to the dataset shows that the two most compositionally

433 associated taxa are Prevotella sp. and Megasphaera sp. Note, that these taxa do not

434 have the shortest links in the compositional biplot, indicating that the amount of variance

435 explained is not high enough to provide an accurate projection of the dataset.

436 For the second worked example we include in the workshop.Rnw document a

437 second example based on the data of Hsiao et al. (2013) that examined the effect of

438 Bacteriodes fragilus supplementation on the microbiome composition of a mouse model

439 of autism. This paper determined that there was a strong functional association between

440 B. fragilus supplementation and mouse behavior. One of the major conclusions was that

441 this functional change in behavior was associated with changes in abundance of a

442 number of bacteria that composed the mouse gut microbiome. We will focus our

443 analysis only on the conclusions derived from the analysis of the microbiome data that

444 were presented in Figure 4 of the paper.

445 Figure 5 shows a compositional biplot of this dataset, and it is obvious that there

446 is little evidence of difference between the poly-IC treated control (IC) and poly-IC

447 treated mice supplemented with $B$. fragilus $(\mathrm{Bf})$ groups when analyzed using this

448 approach. This is in accordance with their conclusions when analyzing the data using

449 an unweighted Unifrac distance based approach. Interestingly, the compositional biplot

450 shows that the $\mathrm{Bf}$ samples are generally closer to the origin of the plot than are the IC

451 samples, suggesting that the $\mathrm{Bf}$ samples have lower dispersion than the IC samples.

$452 \quad$ Since the authors concluded that there was no evidence for multivariate

453 differences between groups, and the CoDa approach agrees, it is generally not advised 
454 to conduct a univariate analysis since it is likely that only false positive results would be 455 obtained (Hubert and Wainer 2012).

$456 \quad$ However, these authors went on to identify a number of univariate differences in

457 taxon abundance between groups using the LEfSe and Metastats tools that are

458 standard in the field (White et al. 2009, Segata et al. 2012), but that do not assume the

459 data are multivariate compositions. When examining univariate differences with the

460 ALDEx2 tool, we found that none of the univariate differences reported in the original

461 paper were supported by subsequent analysis. In particular, the authors indicated that

462 the largest differences between groups were found for six taxa labeled as $53,145,638$,

463836,837 , and 956 in Figure 4 of the paper. The reason for this discrepancy is that

464 inspection of the original paper reveals that raw, and not Benjamini-Hochberg adjusted

$465 P$ values were reported. Thus it is likely that the majority, if not all, of the taxa different

466 between the control and treatment groups are false positive identifications. This result is

467 congruent with the multivariate results found in both the original paper, and by the

468 compositional biplot. Finally, in support of this assertion, we observe that all of these

469 predicted differences become insignificant following a multiple test correction using

470 either the $\mathrm{P}$ values reported in the paper, or $\mathrm{P}$ values calculated using the ALDEx2

471 software.

472 While we have been critical of the microbiome analysis methods used in this

473 paper, we must acknowledge that other published papers exhibit many of the same

474 flaws: namely an over-reliance on tools that do not treat the data as compositions, the

475 identification of extremely rare taxa as the most 'significantly different' taxa between

476 groups, and a general lack of corrections for multiple hypothesis testing. 


\section{Summary}

478 Because the total number of reads is uninformative in high throughput DNA

479 sequencing datasets, the only information available is the ratio of abundances between

480 components: thus these data are compositional. Using two 16S rRNA gene sequencing

481 datasets, we have illustrated that microbiome data can be examined using a

482 multivariate CoDa approach where the data are ratios between the OTU count in a

483 sample and geometric mean for that sample. Dirichlet Monte-Carlo replicates coupled

484 with the centered log-ratio transformation can ameliorate the sparse data problem

485 inherent in microbiome datasets.

486 In essence, we argue here that 16S rRNA gene sequencing datasets are not

487 special and do not need their own unique statistical analysis approaches. The data

488 generated can be examined by a general multivariate approach after accounting for the

489 compositional nature of the data, and such an analysis is comparable or superior to

490 domain-specific approaches, such as those used in the second example paper (Hsiao

491 et al. 2013).

492 With the human body associated with a large number and diversity of bacteria,

493 we need to understand the evolution of this association and how and when this intimate

494 association develops. Such understanding will in turn lead us to robust approaches

495 focussed on when and how to influence the microbiome by probiotic supplementation or

496 by nutrient or antimicrobial means. More and more studies are exploring how the

497 microbiome can predict outcomes, including following fecal transplant, probiotic, dietary

498 and drug treatment (David et al. 2014; Kwak et al. 2014; Seekatz et al. 2014; Rajca et

499 al. 2014). Such work will require carefully designed studies with high quality clinical 
500 documentation, and samples that are processed using some of the methods described

501 herein. As the compositional toolkit for microbiome analysis evolves, these studies will

502 reveal aspects of human life not previously envisaged. In order to have confidence in

503 such findings, datasets must be interrogated with rigour. The public is thirsty for

504 knowledge and the media anxious to attract attention. Reliance on pharmaceutical

505 agents is longer acceptable, and the ability to manipulate the microbiome is not only

506 appealing but actually feasible. Thus, studies that help to understand how such

507 manipulations occur, what communication is taking place between microbes and the

508 host, will allow for more precisely targeted interventions, even to some extent

509 personalized. In particular for the latter, as precise knowledge of microbiome

510 components and activity will be critical.

511 Interested readers wishing to progress beyond this demonstration should consult

512 the compositional data literature, but in particular the original book by Aitchison (1986)

513 and a comprehensive book by Pawlowsky-Glahn et al. (2015) that outlines the essential

514 geometric problem of compositional data as it is understood at present. For a guide that

515 goes beyond the introduction given here and in the supplementary material, a book

516 outlining how to use the compositions R package by Van den Boogaart and Tolosana-

517 Delgado (2013) is particularly helpful, although none of the examples are drawn from

518 the biological literature. For others wishing to understand bioinformatics and data

519 analysis of sequencing data in general terms, hopefully this paper will prove helpful, and

520 encourage people to enroll in specialized courses. The temptation may be to rely on

521 proprietary third party systems, even at a cost, but the 'devil is in the details' and for 
522 thoroughness we recommend developing the highest level of skill possible, especially to 523 continue to create new analytical tools.

524 We hope that this report will help researchers to better understand their data and

525 thereby conduct analyses that are more likely to be robust, and more importantly to 526 bring badly needed breakthroughs in prevention, treatment and cure of disease. 
528 Funding: Financial support for this study was provided by a joint Canadian Institutes of

529 Health Research (CIHR) Emerging Team Grant and a Genome British Columbia (GBC)

530 grant awarded on which GR was a co-PI and GG and ML were co-investigators (grant

531 reference \#108030). Work in the lab of GG is also supported by an NSERC Discovery

532 Grant. The funders had no role in study design, data collection and analysis, decision to 533 publish, or preparation of the manuscript.

534 


\section{References}

536 Aitchison, J. 1986. The statistical analysis of compositional data, Chapman and Hall,

537 London England. ISBN 1-930665-78-4

538 Aitchison, J and Greenacre, M. 2002. Biplots of compositional data. J. Royal Stat. Soc:

539 Series C. 51:375-92

540 Benjamini, Y., Hochberg, Y. 1995. Controlling the false discovery rate: a practical and

541 powerful approach to multiple testing. . Royal Stat. Soc: Series B (Methodological), 289-

542300.

543 David, L.A., Maurice, C.F., Carmody, R.N., Gootenberg, D.B., Button, J.E., Wolfe, B.E.,

544 Ling, A.V., Devlin, A.S., Varma, Y., Fischbach, M.A., Biddinger, S.B., Dutton, R.J.,

545 Turnbaugh, P.J. 2014. Diet rapidly and reproducibly alters the human gut microbiome.

546 Nature. 505(7484):559-63.

547 Di Bella, J.M., Bao, Y., Gloor, G.B., Burton, J.P., Reid, G. 2013. High throughput

548 sequencing methods and analysis for microbiome research. J Microbiol Methods. 2013

549 Dec;95(3):401-14.

550 Fernandes, A. D., Macklaim, J. M., Linn, T. G., Reid, G., Gloor, G. B. 2013. ANOVA-like

551 differential expression (ALDEx) analysis for mixed population RNA-Seq. PloS One, 8(7),

552 e67019.

553 Fernandes, A.D., Reid, J.N., Macklaim, J.M., McMurrough, T.A., Edgell, D.R., Gloor,

554 G.B. 2014. Unifying the analysis of high-throughput sequencing datasets: characterizing

555 RNA-seq, 16S rRNA gene sequencing and selective growth experiments by

556 compositional data analysis. Microbiome. 2:15. 
557 Filzmoser, P., Hron, K., Reimann, C. 2009. Univariate statistical analysis of

558 environmental (compositional) data: problems and possibilities. Sci tTtal Environ.

$559 \quad 407: 6100-8$.

560 Frémont, M., Coomans, D., Massart, S., De Meirleir, K.. 2013. High-throughput 16 S

561 rRNA gene sequencing reveals alterations of intestinal microbiota in myalgic

562 encephalomyelitis/chronic fatigue syndrome patients. Anaerobe. 22:50-6.

563 Friedman, J., Alm, E. J. 2012. Inferring correlation networks from genomic survey data.

564 PLoS Comput. Biol. 8(9): e1002687

565 Gentleman, R. C., Carey, V. J., Bates, D. M., Bolstad, B., Dettling, M., Dudoit, S., Ellis,

566 B. et al. 2004. Bioconductor: open software development for computational biology

567 and bioinformatics. Gen. Biol. 5 (10): R80.

568 Gloor, G.B., Macklaim, J.M., Fernandes, A.F. 2016. Displaying variation in large

569 datasets: a visual summary of effect sizes. J. Comput. Graph. Stat. (in press),

570 DOI:10.1080/10618600.2015.1131161.

571 Gloor, G.B., Macklaim, J.M., Vu, M, Fernandes, A.F. 2016. Compositional uncertainty

572 should not be ignored in high-throughput sequencing data analysis. Austrian Journal of

573 Statistics (in press).

574 Gloor, G.B. 2016. Compositional data analysis for high throughput sequencing: an

575 example from 16S rRNA gene sequencing. Supplementary Information at:

576 http://github.com/ggloor/CJM Supplement. DOI:10.5281/zenodo.49579.

577 Hsiao, E. Y., McBride, S.W., Hsien, S., Sharon, G., Hyde, E.R., McCue, T., Codelli, J.A.,

578 Chow, J., Reisman, S.E., Petrosino, J.F., Patterson, P.H., Mazmanian,S.K. 2013. 
579 Microbiota modulate behavioral and physiological abnormalities associated with

580 neurodevelopmental disorders. Cell. 155(7):1451-63

581 Hubert, L., Wainer, H. 2012. A statistical guide for the ethically perplexed. CRC Press,

582 London, UK.

583 Kuczynski, J., Stombaugh, J., Walters, W. A., González, A., Caporaso, J. G., Knight, R.

584 2012. Using QIIME to analyze 16S rRNA gene sequences from microbial communities.

585 Curr. Prot. Microbiol. 1E-5.

586 Kurtz, Zachary D and Müller, Christian L and Miraldi, Emily R and Littman, Dan R and

587 Blaser, Martin J and Bonneau, Richard A 2015. Sparse and compositionally robust

588 inference of microbial ecological networks. PLoS Comp. Bio. 11:e1004226

589 Kwak, D.S., Jun, D.W., Seo, J.G., Chung, W.S., Park, S.E., Lee, K.N., Khalid-Saeed,

590 W., Lee, H.L., Lee, O.Y., Yoon, B.C., Choi, H.S. 2014. Short-term probiotic therapy

591 alleviates small intestinal bacterial overgrowth, but does not improve intestinal

592 permeability in chronic liver disease. Eur J Gastroenterol Hepatol. 26(12):1353-9.

593 Lourenço, T.G., Heller, D., Silva-Boghossian, C.M., Cotton, S.L., Paster, B.J., Colombo,

594 A.P. 2014. Microbial signature profiles of periodontally healthy and diseased patients. J

595 Clin Periodontol. 41(11):1027-36.

596 Lovell, D., Pawlowsky-Glahn, V., Egozcue, J. J. Marguerat, S., Bähler, J. 2015.

597 Proportionality: a valid alternative to correlation for relative data. PLoS Comput Biol

$598 \quad 11: \mathrm{e} 1004075$.

599 Lozopone, C., Knight, R. 2005. Unifrac: a new phylogenetic method for comparing

600 microbial communities. Applied Env. Micro. 71:8228-8235. 
601 Macklaim, J.M., Clemente, J.C., Knight, R., Gloor, G.B., Reid, G. 2015. Changes in 602 vaginal microbiota following antimicrobial and probiotic therapy. Microb Ecol Health Dis. $60326: 27799$.

604 Mandal, S., Van Treuren, W., White, RA., and Eggesbø, M., Knight, R., Peddada, S. D. 605 2015. Analysis of composition of microbiomes: a novel method for studying microbial 606 composition. Microl. Ecol. Health Dis. 26:27663.

607 Martín-Fernández, J. A., Barceló-Vidal, C., \& Pawlowsky-Glahn, V. 1998. Measures of 608 difference for compositional data and hierarchical clustering methods. In A. Buccianti, 609 G. Nardi, \& R. Potenza (Eds.), Proc. IAMG (Vol. 98, pp. 526-531).

610 Palarea-Albaladejo J., Antoni Martín-Fernández, J. 2015. zCompositions --- R package 611 for multivariate imputation of left-censored data under a compositional approach.

612 Chemometrics and Intelligent Laboratory Systems. 143:85-96

613 Pawlowsky-Glahn, V., Egozcue, J. J., Tolosana-Delgado, R. 2015. Modeling and

614 Analysis of Compositional Data. John Wiley \& Sons. Springer. 258 pg, London, UK.

615 Pearson, K. 1896. Mathematical contributions to the theory of evolution. -- on a form of 616 spurious correlation which may arise when indices are used in the measurement of 617 organs. Proc. Royal Soc. Lond. 60:489-498

618 R Core Team 2015. R: A language and environment for statistical computing. $R$

619 Foundation for Statistical Computing, Vienna, Austria. URL https://www.R-project.org/.

620 Rajca, S., Grondin, V., Louis, E., Vernier-Massouille, G., Grimaud, J.C., Bouhnik, Y.,

621 Laharie, D., Dupas, J.L., Pillant, H., Picon, L., Veyrac, M., Flamant, M., Savoye, G.,

622 Jian, R., Devos, M., Paintaud, G., Piver, E., Allez, M., Mary, J.Y., Sokol, H., Colombel, 623 J.F., Seksik, P. 2014. Alterations in the intestinal microbiome (dysbiosis) as a predictor 
624 of relapse after infliximab withdrawal in Crohn's disease. Inflamm Bowel Dis. 20(6):97862586.

626 Reardon, S. 2013, Bacterium can reverse autism-like behaviour in mice. Nature.

627 doi:10.1038/nature.2013.14308.

628 Schellenberg, J., Links, M. G., Hill, J. E., Dumonceaux, T. J., Peters, G. A., Tyler, S.,

629 Ball, T. B., Severini, A., Plummer, F. A. 2009. Pyrosequencing of the chaperonin-60

630 universal target as a tool for determining microbial community composition. Appl

631 Environ Microbiol. 75: 2889-98.

632 Schloss, P.D, Westcott, S.L, Ryabin, T., Hall, J.R., Hartmann, M., Hollister, E.B.,

633 Lesniewski, R.A., Oakley, B.B., Parks, D.H., Robinson, C.J., Sahl, J.W., Stres, B.,

634 Thallinger, G.G., and Van Horn, D.J., Weber, C.F. 2009. Introducing mothur: open-

635 source, platform-independent, community-supported software for describing and

636 comparing microbial communities

637 Seekatz, A.M., Aas, J., Gessert, C.E., Rubin, T.A., Saman, D.M., Bakken, J.S., Young,

638 V.B. 2014. Recovery of the gut microbiome following fecal microbiota transplantation.

639 MBio. 5(3):e00893-14.

640 Segata, N., Izard, J., Waldron, L., Gevers, D., Miropolsky, L., Garrett, W.S.,

641 Huttenhower, C. 2011. Metagenomic biomarker discovery and explanation. Genome

642 Biol. 12:R60

643 Urbaniak, C., Cummins, J., Brackstone, M., Macklaim, J.M., Gloor, G.B., Baban, C.K.,

644 Scott, L., O'Hanlon, D.M., Burton, J.P., Francis, K.P., Tangney, M., Reid, G. 2014.

645 Microbiota of human breast tissue. Appl Environ Microbiol. 80(10):3007-14. Van den 
646 Boogaart, K. G., Tolosana-Delgado, R. 2013. Analyzing compositional data with R.

647 Heidelberg: Springer. Heidelberg 258 pages.

648 Walker, A. W., and Martin, J.C., Scott, P., Parkhill, J., Flint, H. J. Scott, K. P. 2015. 16 S

649 rRNA gene-based profiling of the human infant gut microbiota is strongly influenced by

650 sample processing and PCR primer choice. Microbiome. 3:26

651 Warton, D.I., Wrigth, S.T., Wang, Y. 2012. Distance-based multivariate analyses

652 confound location and dispersion effects. Methods Ecol. Evol. 3:89-101.I

653 White, J.R., Nagarajan, N., Pop, M. 2009. Statistical methods for detecting differentially

654 abundant features in clinical metagenomic samples. PLoS Comput. Biol. 5:e1000352

655

656 


\section{Figure Legends}

658 Figure 1: The difference between counting, proportions and ratios. The 'Counts' panel

659 shows a scatter plot of a simulated dataset with two samples composed of 49 invariant

660 taxa in open circles, and 1 taxon that changes in count 10-fold (black-filled circle). This

661 is the type of data that most current analysis tools in the microbiome field expect is

662 being analyzed. The 'Proportions' panel shows the same samples after they have been

663 sequenced and so constrained to have a constant sum. With such a constraint, their

664 representation is the same whether the sum is 1 (as shown here) or an arbitrarily larger

665 number (such as would be obtained from a sequencing instrument). The distortion in the

666 data is obvious: the black-filled circle still appears to be more abundant, but the open

667 circles appear to have become less abundant! It is obvious that we would draw incorrect

668 inferences regarding abundance changes in these data, yet these are the data as used

669 by existing tools. The third panel shows that much of this distortion can be removed

670 using a ratio transformation where each count (or proportion) is divided by the

671 geometric mean of the 50 taxa in the sample. Examination of the data after this

672 transformation can thus provide more robust inferences.

673 Figure 2: The left figure shows a covariance biplot of the abundance-filtered dataset,

674 the right figure shows a scree plot of the same data. This exploratory analysis is

675 encouraging, but not definitive, because the amount of variance explained is substantial

676 with 0.469 of the variance being explained by component 1 , and 0.139 being explained

677 by component 2 . The numbers on the left and right indicated unit-scaled variance of the

678 taxa, the numbers on the top and right indicate unit scaled variances of the samples.

679 Samples are colored in red if diagnosed as BV, blue if healthy, and purple if 
680 intermediate. The scree plot also shows that the majority of the variability is on

681 component 1 . We can interpret this biplot with some confidence, although it is likely that 682 any associations will be found to have large variation.

683 Figure 3: Unsupervised clustering of the reduced dataset. The top figure shows a 684 dendrogram of relatedness generated by unsupervised clustering of the Aitchison 685 distances, which is a distance that is robust to perturbations and sub-compositions of 686 the data (Aitchison 1986). The bottom figure shows a stacked bar plot of the samples in 687 the same order. The legend indicating the colour scheme for the taxa is on the right side.

688 Figure 4: An effect plot showing the univariate differences between groups (Gloor et al. 689 2015). The left plot shows a plot of the maximum variance within the B or A group vs.

690 the difference between groups. Large black points indicate those that have a mean 691 Benjamini-Hochberg adjusted P-value of 0.05 or less using P values calculated with the 692 Wilcoxon rank test. The middle plot shows a plot of the effect size vs. the adjusted P 693 value. In general, effect size measures are more robust than are $P$ values and are 694 preferred. For a large sample size such as this one, an effect size of 0.5 or greater will 695 likely correspond to biological relevance. The right plot shows a volcano plot where the 696 difference between groups is plotted vs the adjusted $P$ value.

697 Figure 5: A form biplot of the Hsiao et al. (2013) dataset that best represents the 698 distances between samples. Here we can see that the control and experimental 699 samples are intermingled, suggesting no separation between the groups. Furthermore, 700 the proportion of variance explained in the first component is not large when compared 701 to the other components. The evidence of structure within this dataset is thus weak. 
703 Table 1: List of significantly different taxa.

\begin{tabular}{lllllll} 
Taxon & diff.btw & diff.win & effect & overlap & wi.ep & wi.eBH \\
\hline Atopobium & 0.86 & 1.51 & 0.53 & 0.30 & 0.007 & 0.037 \\
Prevotella & 1.41 & 1.77 & 0.75 & 0.22 & 0.000 & 0.002 \\
L. crispatus & -1.07 & 1.78 & -0.49 & 0.23 & 0.000 & 0.004 \\
L. iners & -2.25 & 2.68 & -0.79 & 0.20 & 0.000 & 0.001 \\
Streptococcus & -1.14 & 2.38 & -0.37 & 0.30 & 0.008 & 0.041 \\
Dialister & 0.89 & 1.38 & 0.59 & 0.25 & 0.001 & 0.009 \\
Megasphaera & 1.56 & 2.31 & 0.63 & 0.28 & 0.002 & 0.015
\end{tabular}

704 diff.btw: median difference between groups on a log base 2 scale

705 diff.win: largest median variation within group H or BV

706 effect: effect size of the difference, median of diff.btw/diff.win

707 overlap: confusion in assigning an observation to $\mathrm{H}$ or BV group. Smaller is better

708 wi.ep: expected value of the Wilcoxon Rank Test P-value

709 wi.eBH: expected value of the Benjamini-Hochberg corrected P-value

710

711 


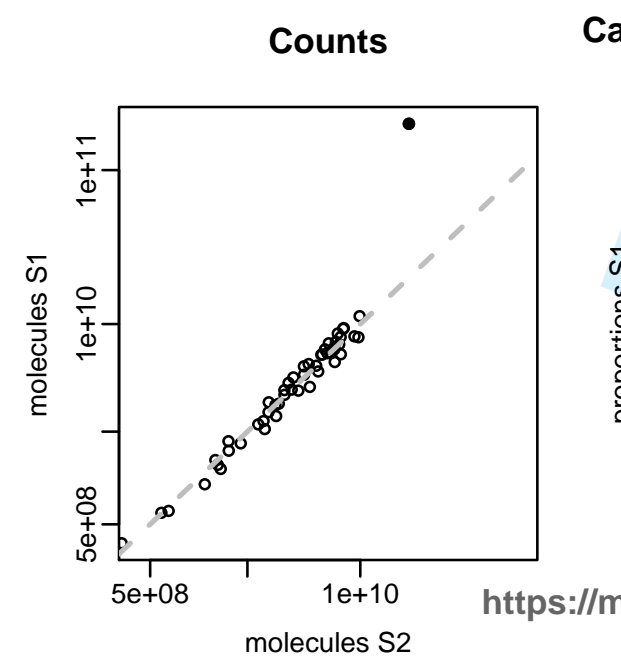

Canadian Joupläp8frMigrobiology

Ratiage 36 of 40
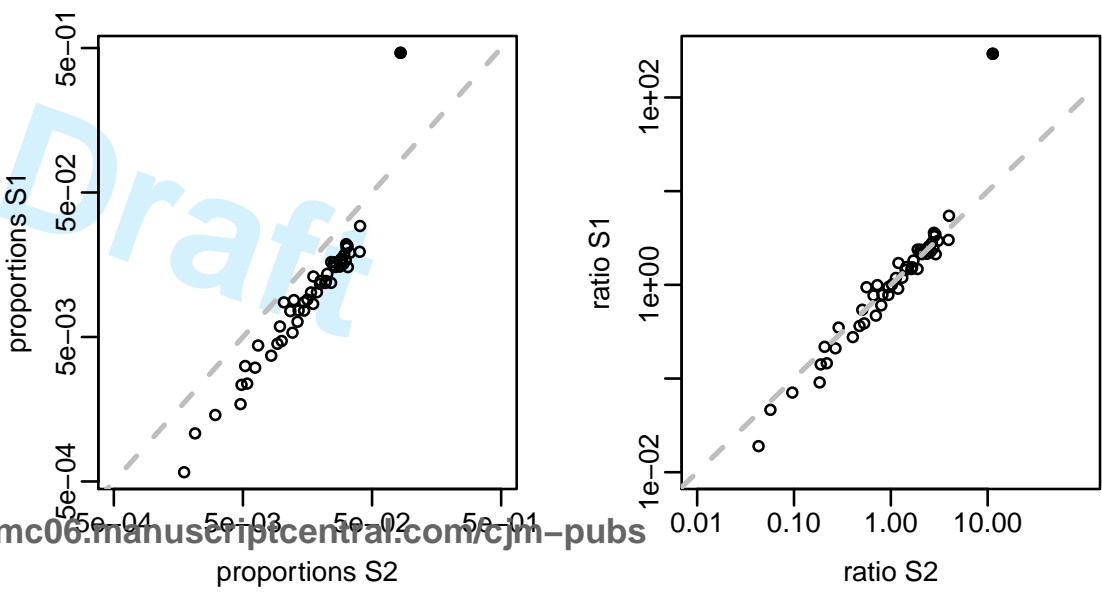
Canadian Journal of Microbiology

Biplot

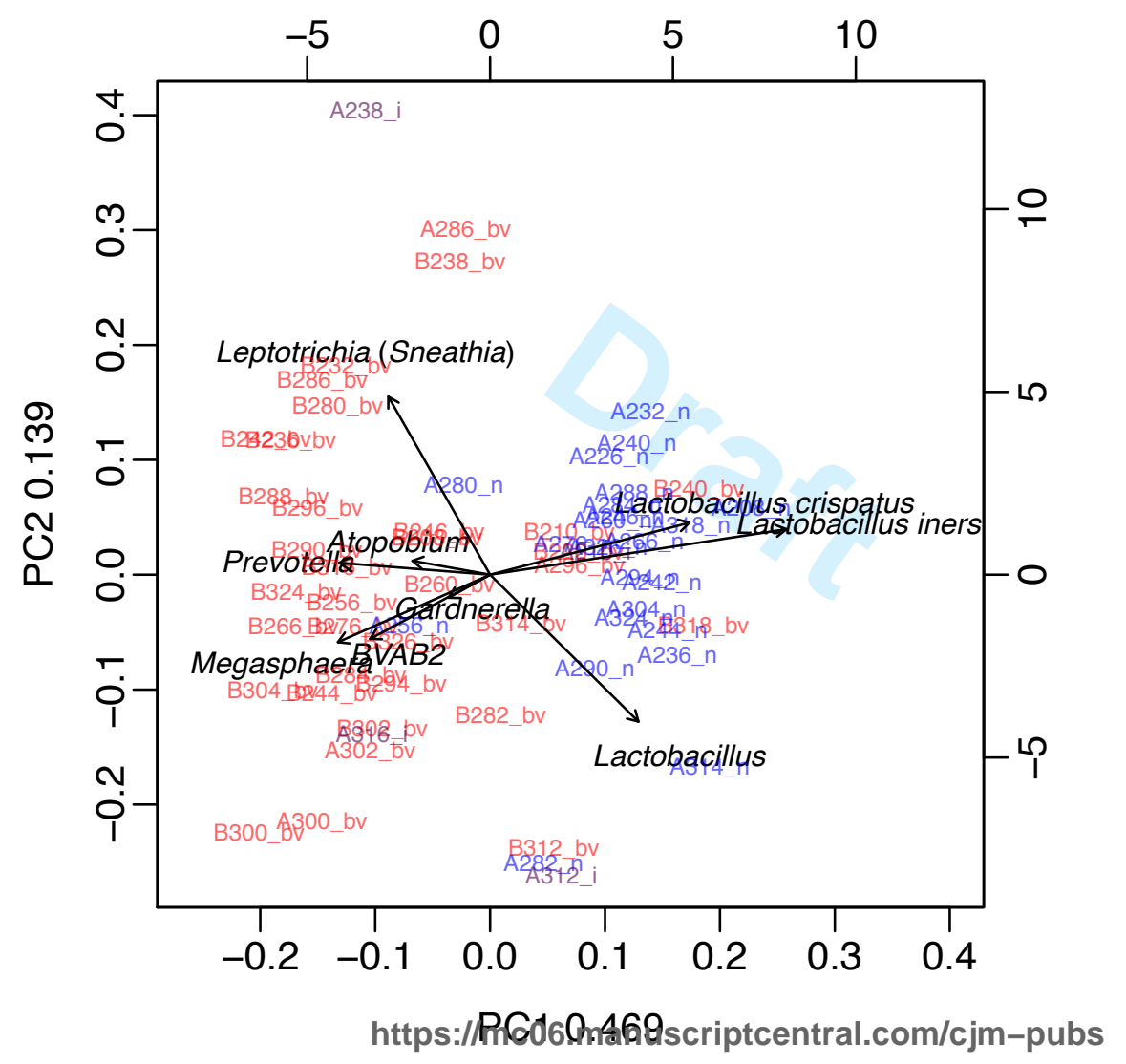

\section{Scree plot}

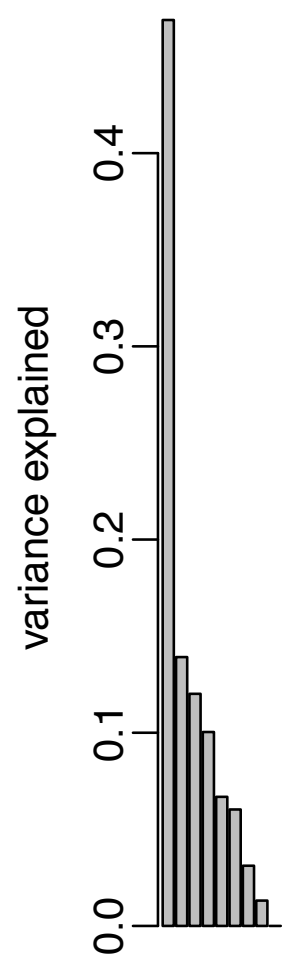

Component 

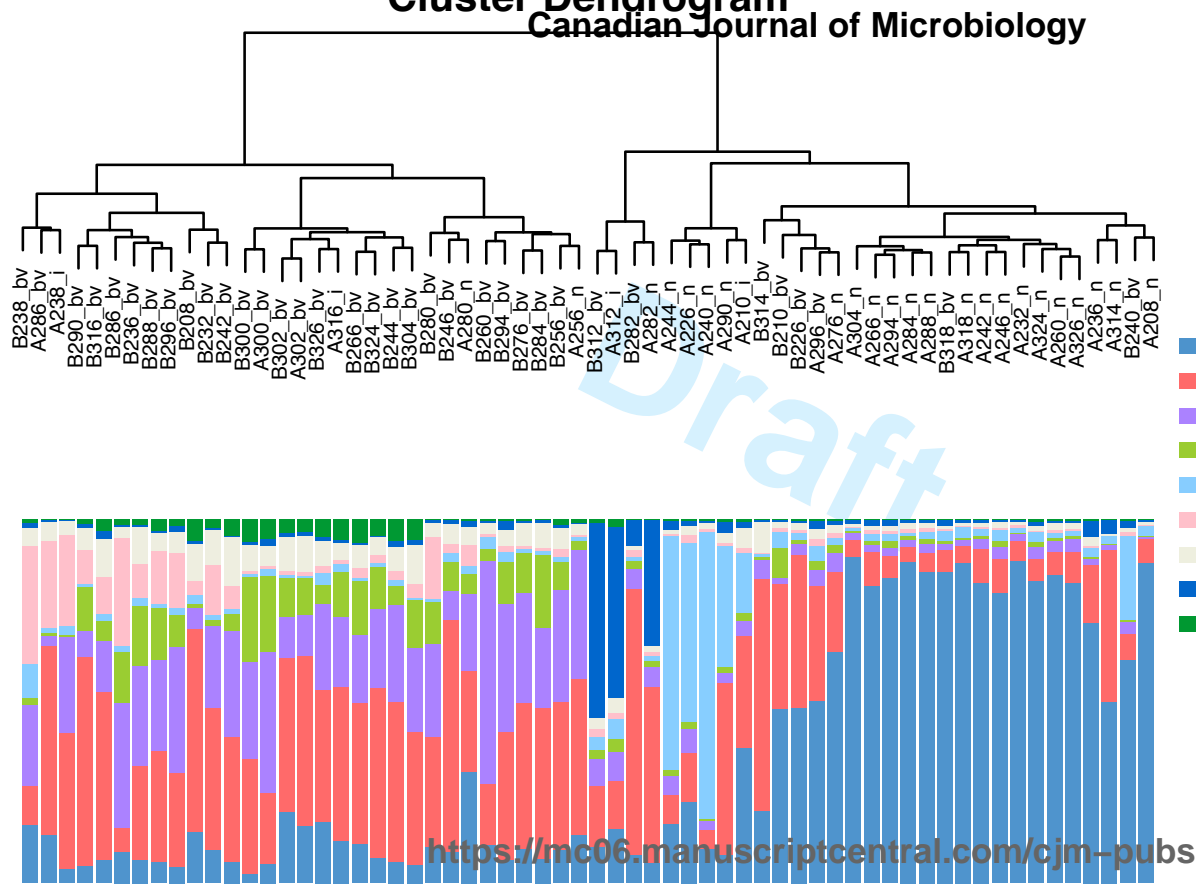

Firmicutes:Lactobacillus iners Actinobacteria:Gardnerella Bacteroidetes:Prevotella Firmicutes:Megasphaera Firmicutes:Lactobacillus crispatus Fusobacteria:Leptotrichia Actinobacteria:Atopobium Firmicutes:Lactobacillus Firmicutes:BVAB2 

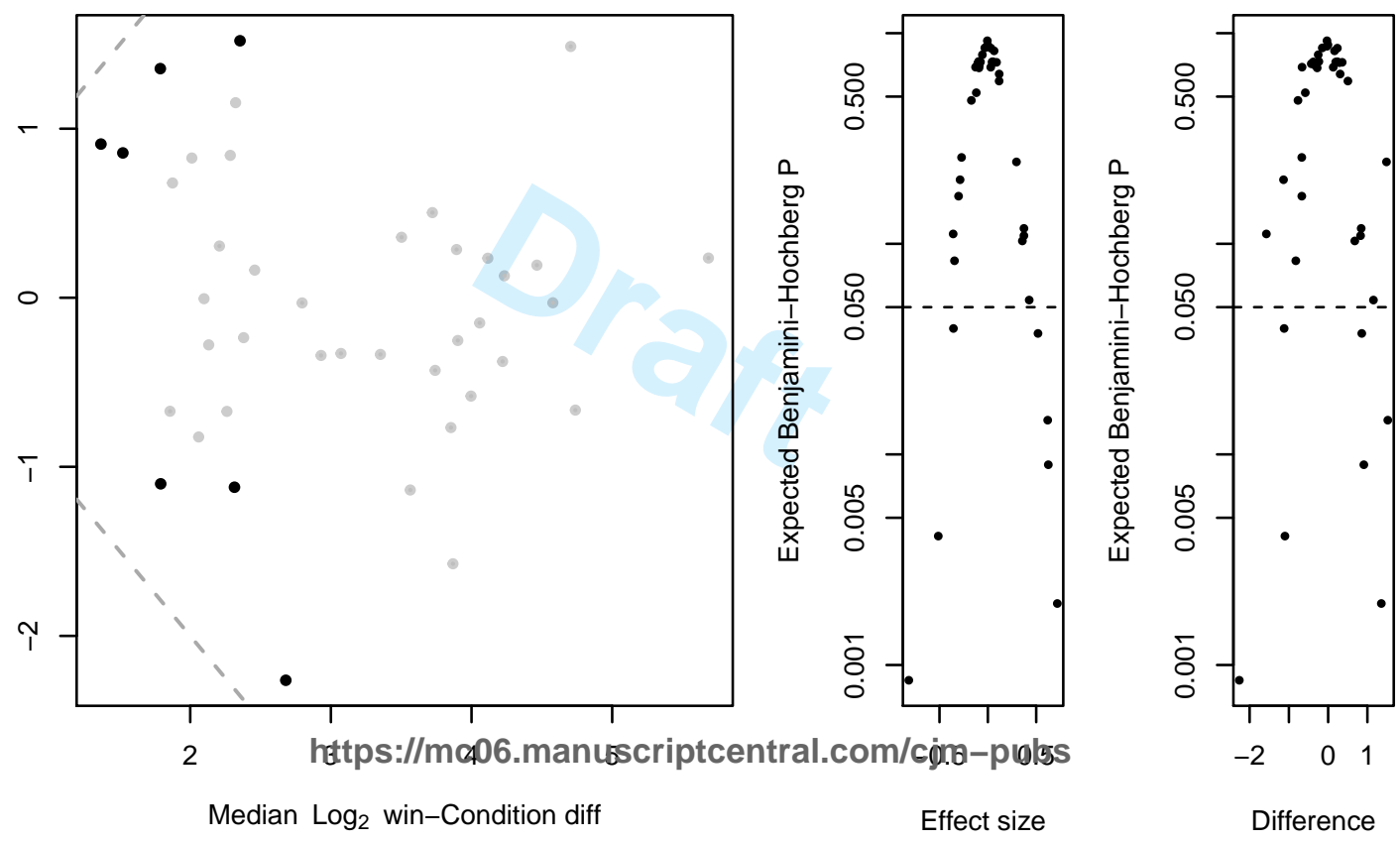

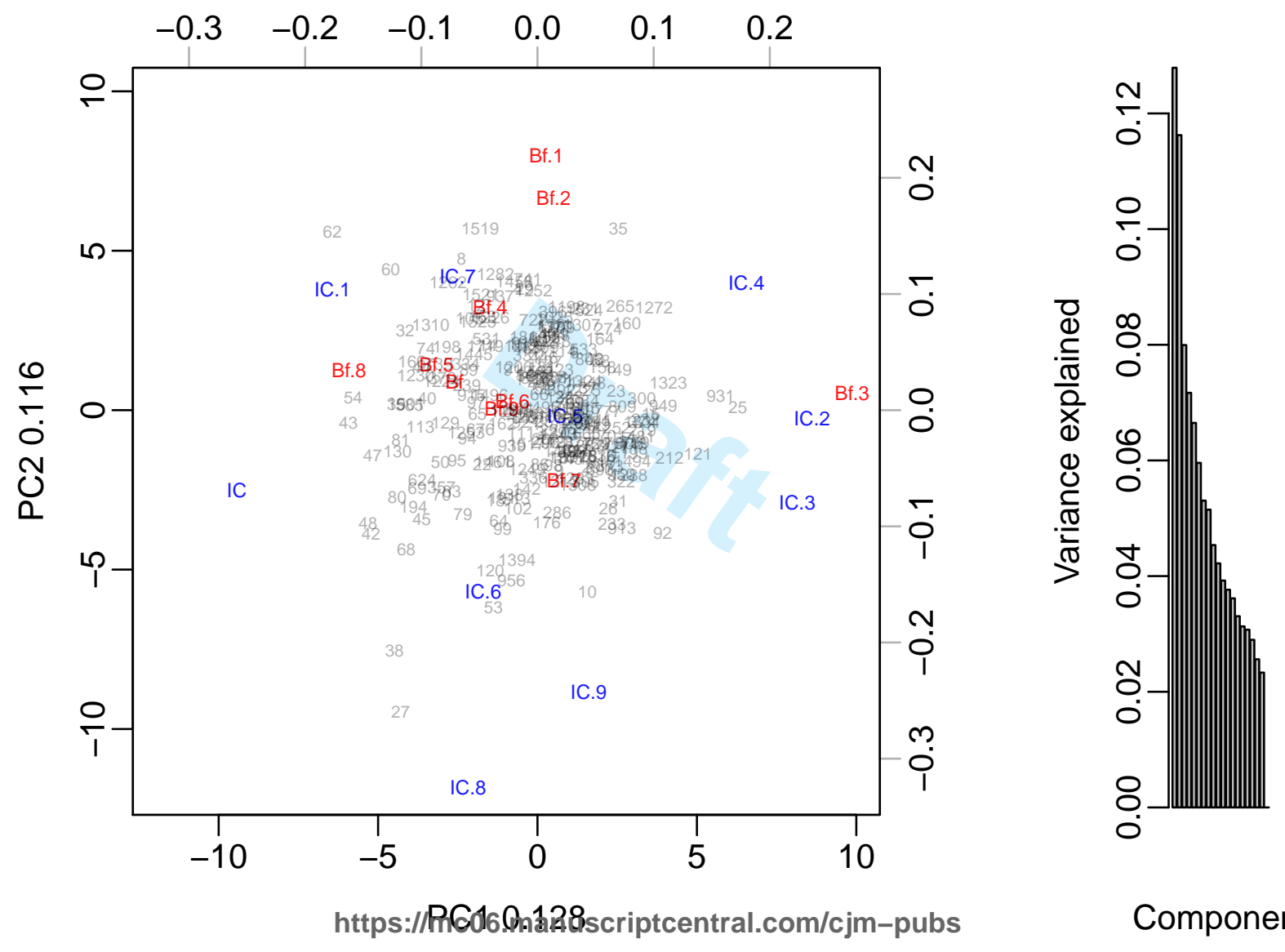

Component 